\title{
Predicting outcome in mild cognitive impairment: 4-year follow-up study
}

Jane A. Lonie, Mario A. Parra-Rodriguez, Kevin M. Tierney, Lucie L. Herrmann, Claire Donaghey, Ronan E. O'Carroll and Klaus P. Ebmeier

\section{Background}

Cognitive impairment precedes the diagnosis of Alzheimer's disease. It is unclear which psychometric measures predict dementia, and what cut-off points should be used. Replicable cognitive measures to provide information about differential diagnosis and prognosis would be clinically useful.

\begin{abstract}
Aims
In a prospective cohort study we investigated which measures distinguish between individuals with amnestic mild cognitive impairment (aMCl) that converts to dementia and those whose impairment does not, and which combination of measures best predicts the fate of people with aMCl.
\end{abstract}

\section{Method}

Forty-four participants with aMCl underwent extensive neuropsychological assessment at baseline and annually thereafter for an average of 4 years. Differences in baseline cognitive performance of participants who were converters and non-converters to clinically diagnosed dementia were analysed. Classification accuracy was estimated by sensitivity, specificity, positive and negative predictive values and using logistic regression.

\section{Results}

Forty-one percent of participants had progressed to dementia by the end of study, with a mean annual conversion rate of $11 \%$. Most (63\%) showed persisting or progressive cognitive impairment, irrespective of diagnosis. The Addenbrooke's Cognitive Examination together with the discrimination index of the Hopkins Verbal Learning Test Revised (but none of the demographic indices) differentiated the participants who were converters from the nonconverters at baseline with $74 \%$ accuracy.

\section{Conclusions}

Targeted neuropsychological assessment, beyond simple cognitive screening, could be used in clinical practice to provide individuals with $\mathrm{aMCl}$ with prognostic information and aid selective early initiation of monitoring and treatment among those who progress towards a clinically diagnosable dementia.

\section{Declaration of interest}

None.
Criteria for amnestic mild cognitive impairment (aMCI) have been devised in an attempt to capture the preclinical phase of Alzheimer's dementia. There is evidence to suggest, however, that individuals with aMCI according to the current criteria comprise a heterogeneous group, ${ }^{1,2}$ some of whom will progress to dementia with time, while others will not. ${ }^{3}$ To maximise the diagnostic value of aMCI, criteria should identify a homogeneous group of people with preclinical dementia. To this end, cognitive criteria should be defined in a manner that reflects our current knowledge of their predictive value. As the prodromal phase of Alzheimer's disease is likely to extend beyond a 20 -year period, ${ }^{4}$ studies with shorter follow-up periods are liable to underestimate the risk of conversion. In fact, only 2 of 14 clinic-based longitudinal aMCI studies report follow-up periods beyond 3 years (online Table DS1). ${ }^{5,6}$ Moreover, low baseline levels of general cognitive functioning among participants with aMCI lead to a greater chance of neuropsychological tasks predicting dementia, because of the more advanced stage of disease in the cohort with aMCI. In 11 of 14 clinic-based longitudinal studies average baseline Mini-Mental State Examination (MMSE) ${ }^{7}$ scores for the group with mild cognitive impairment who converted to dementia (aMCI converters) fell below the higher screening cut-off for dementia (i.e. 27/30). In these studies, an underlying dementia might well have been suspected on the basis of such rudimentary cognitive screening instruments alone, begging the question of the 'added value' of a fuller cognitive evaluation. The comprehensiveness of the neuropsychological battery employed and the appropriateness (on both theoretical and empirical grounds) of test selection might also be expected to influence predictive power.
For example, the combination of the Paired Associate Learning test (PAL), ${ }^{8}$ age and the Graded Naming Test $(\mathrm{GNT})^{9}$ give an overall classification accuracy of $100 \%$ over a 2.5 year follow-up interval. ${ }^{8}$ The same classification accuracy (i.e. 100\%) has been reported $^{10}$ for the use of the PAL in combination with the Addenbrooke's Cognitive Examination $(\mathrm{ACE})^{11}$ and the Graded Faces Test $(\mathrm{GFT})^{12}$ over shorter (1 year) intervals. However, these findings have not been replicated outside the test authors' group, in larger numbers of people with aMCI, across follow-up periods extending beyond 2.5 years, and where the mean general level of cognitive functioning at baseline (as indicated by performance on cognitive screening) falls above cut-off points for dementia. If replicable, such measures could be used in the neuropsychological assessment of aMCI within specialist memory clinic settings to provide information about differential diagnosis and prognosis.

Measurement of cognitive function represents just one, albeit an important, approach to detecting and diagnosing Alzheimer's disease at a very early and preclinical stage. Other work has looked at the ability of imaging (for the most part magnetic resonance image (MRI) scanning), biomarkers (i.e. total tau, beta amyloid 42 and phosphorylated tau) and changes of a behavioural nature to predict the future onset of clinically diagnosable Alzheimer's disease. A recent meta-analysis of imaging and biomarkers for Alzheimer's disease ${ }^{13}$ indicated some promise for the cerebrospinal fluid (CSF) markers in so far as their overall predictive accuracy levels were similar to that of memory impairment 4 years prior to the point of diagnosis. Furthermore, the effect sizes for the CSF markers were largest when assessed longer before the point of diagnosis. However, atrophy of the hippocampus or other 
medial temporal lobe structures was found to be a less accurate predictor of future Alzheimer's disease than memory impairment, and the largest effect sizes, which are themselves likely to represent an underestimation owing to the removal of variability inherent in the inclusion of memory impairment as a selection criterion for a majority of studies, were seen in association with measures of delayed memory recall.

In this study we present a detailed neuropsychological and clinic-based cohort study, with an average of 4 years follow-up from baseline neuropsychological assessment until final review. It is the only study to date of people who can be classified as high-functioning aMCI converters (i.e. MMSE > 27/30) extending beyond 3 years follow-up. Furthermore, it represents the first clinic-based study to investigate the robustness of the GNT, GFT and a combination of the ACE and PAL as predictors of conversion to dementia outside the original authors' research group, ${ }^{11}$ and to report the detailed fate of people who are aMCI non-converters in terms of their course of cognitive impairment.

\section{Method}

\section{Recruitment}

Participants were recruited from the Edinburgh Older Adult Neuropsychology Service, which takes all tertiary referrals over 60 years from geriatricians and old age psychiatrists in the Lothian Region of Scotland. As there is no substantial private sector, these National Health Service (NHS) referrals are likely to be representative of individuals with memory complaints attending their doctor. During the study period from September 2004 to September 2007, 71 people were referred from old age psychiatry, 16 from geriatric medicine. Of these 87,41 did not respond to the invitation to attend or refused to participate in the study, leaving 46 participants. Further details regarding the demographic characteristics of these individuals may be found in a previous publication. ${ }^{14}$

\section{Procedure}

Individuals who fulfilled criteria for $\mathrm{aMCI}^{15}$ (objective cognitive impairment was defined by a performance of 1 standard deviation or more below age means on two or more measures assessing a single cognitive domain) undertook an extensive battery of neuropsychological measures at baseline and were followed up annually, regardless of whether or not they received a clinical diagnosis of dementia during the course of the study, over an average 4-year period. A 1 standard deviation cut-off point on two or more episodic memory measures was used, in place of the more commonly applied 1.5 standard deviations on one or more measures, in an attempt to minimise the likelihood of including participants with aMCI but with an unstable aMCI diagnosis, as well as to maximise sensitivity to memory deficits within our sample with IQs higher than the average participant with aMCI. At the end of the study period participants with aMCI were grouped in accordance with whether or not they had received a clinical diagnosis of dementia (as documented in their medical file) at any point subsequent to their initial study assessment.

A total of 24 age- and IQ-matched healthy elderly participants also completed the full battery of 18 neuropsychological tasks providing a normative comparison group. Sixteen of these 24 healthy participants repeated the battery in full, an average of 28 months later. The retest data were used to established cut-off values and criteria for further classifying the neuropsychological performance of the participants who were aMCI non-converters as 'stable aMCI', 'progressive aMCI', or 'normal' at the study end-point.

\section{Materials, participants and outcome criteria}

Participant characteristics (inclusion/exclusion criteria) and neuropsychological measures have been detailed previously. ${ }^{14,16,17}$ In brief, the neuropsychological battery comprised measures of premorbid IQ (National Adult Reading Test; NART) ${ }^{18}$ episodic memory (PAL, ${ }^{8}$ Hopkins Verbal Learning Test - Revised (HVLT-R), ${ }^{19}$ Rey Complex Figure Test (RCFT), ${ }^{20}$ semantic memory (GFT, GNT, Category fluency; animals), visuospatial function (RCFT copy), psychomotor processing speed (Trail Making Test Part A) ${ }^{21}$ and attention/executive function (Dual Task, ${ }^{22}$ Controlled Oral Word Association Test (COWAT: F, A and S); ${ }^{23}$ Trail Making Test Part B). ${ }^{21}$ Amnestic mild cognitive impairment was defined in accordance with the revised criteria set out by Petersen et al. ${ }^{15}$ Demographic characteristics of the aMCI converters group, the aMCI non-converters group and the normative sample group, together with their respective baseline mean performances on cognitive screening and selected neuropsychological measures, are summarised in Table 1.

\section{Statistical analysis}

Independent $t$-tests were conducted to compare the baseline performances of the aMCI converters group and the aMCI non-converters group on the demographic indices of age, NART full-scale IQ (FSIQ), and years of follow-up and on the neuropsychological measures of ACE total score, PAL, HVLT-R delayed recall and discrimination index (a measure of accuracy of delayed recognition), GFT, Category fluency and Trail Making Test Part B. The alpha level was adjusted to control for multiple comparisons using Holm's sequential Bonferroni correction method. ${ }^{24}$

Baseline ages, time of follow-up and premorbid IQ were selected as potential confounders for their established influence on risk of developing late-onset dementia. ${ }^{25-27}$ Seven neuropsychological measures (ACE, PAL, HVLT-R discrimination index and delayed recall, GNT, GFT and Trail Making Test Part B) were selected from a total of 18 , on the basis of their known sensitivity to aMCI relative to other measures of cognitive functions established by our own cross-sectional analyses, or of their high levels of predictive validity as established by one or more clinic-based longitudinal study of neuropsychological predictors of dementia (online data supplement). Participants who were clinically diagnosed as having dementia at study end-point were identified. Participants who had not received a clinical diagnosis of dementia were further classified as 'normal' or having 'persisting aMCI' based on their neuropsychological profile at end-point, and 'progressive' or 'non-progressive' based on the longitudinal course of cognitive function during their years of study participation. 'Abnormal' neuropsychological performance was defined by a performance at the 7th centile or lower in two or more of the 18 neuropsychological tasks (this would occur by chance in approximately 1 of 22 participants with mild cognitive impairment without a diagnosis of dementia). Mild cognitive impairment decline was defined by cognitive deterioration of a magnitude seen in fewer than $2.5 \%$ of a sample of healthy elderly people over an average 28 -month period on at least two measures of semantic memory or executive functioning. Cognitive domains other than episodic memory were selected for this criterion, because of the baseline floor-level performances on episodic memory tasks of many participants with aMCI.

Sensitivity, specificity, positive and negative predictive values, together with the overall percentage of classification accuracy in predicting conversion or non-conversion to dementia, were determined using a combination of a total score of $<88 / 100$ on the ACE or a performance of 2 standard deviations or more below 
Table 1 Demographic and baseline neuropsychological data for healthy elderly control group, group with amnestic mild cognitive impairment (aMCl) who converted to dementia and group with aMCl who did not convert

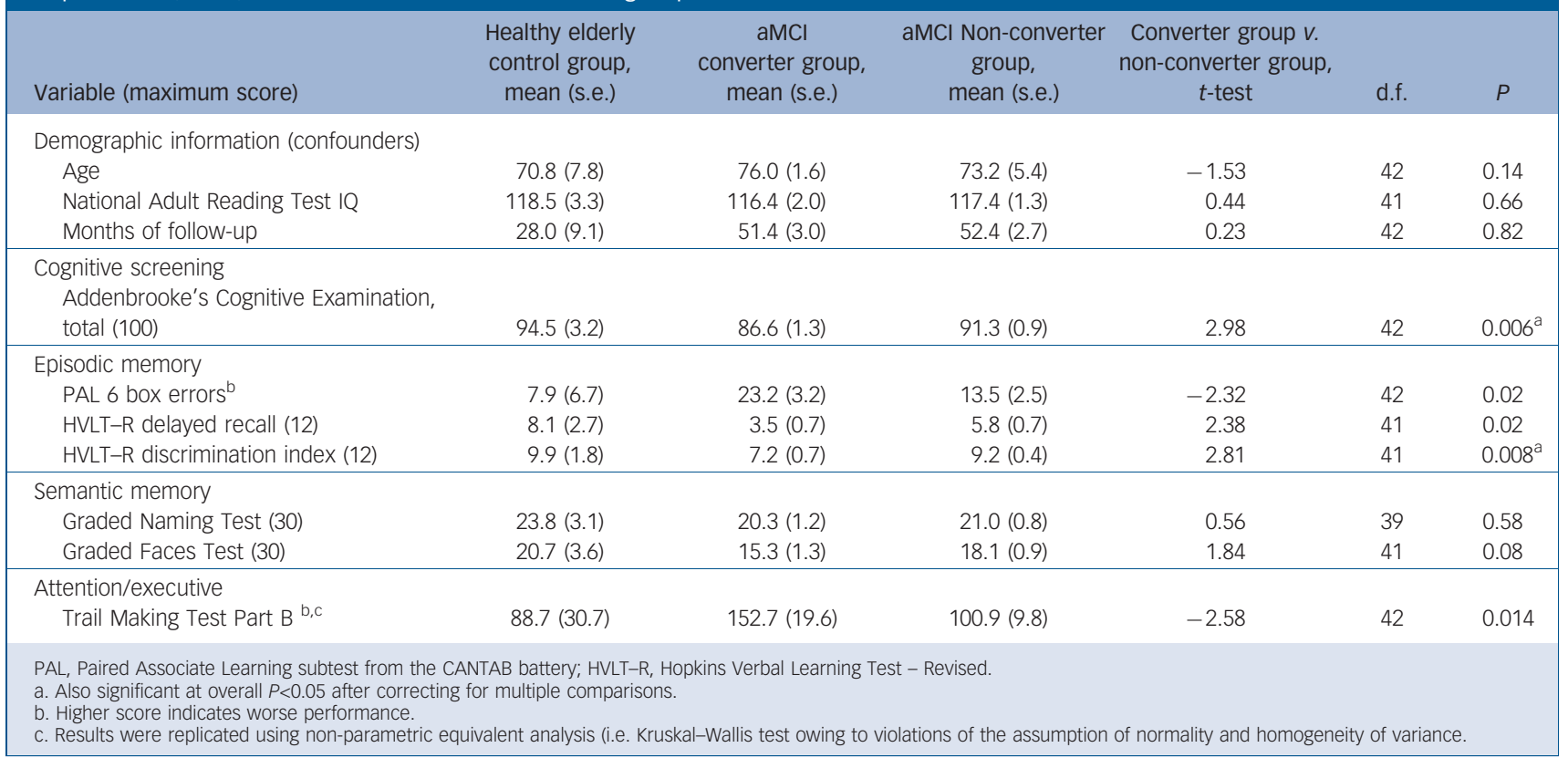

controls on the PAL, as this combination of measures has previously been associated with $100 \%$ sensitivity and negative predictive values. ${ }^{10}$ These values were also determined for face (GFT) and object (GNT) naming measures.

Neuropsychological measures, for which the baseline performances of those in the aMCI converters group and the aMCI non-converters group were significantly different, were entered simultaneously alongside the putative confounders 'age', 'NART FSIQ' and 'years of follow-up' into a logistic regression analysis. A backward stepwise procedure using the likelihood ratio was applied to determine model content and levels of overall classification accuracy. Criteria for entry and removal were set at
$P=0.05$ and $P=0.01$, respectively, using 20 iterations (SPSS 17 for Windows).

\section{Results}

Forty-one percent (18/44, 95\% CI 28-56\%) of participants with aMCI received a clinical diagnosis of dementia (most often Alzheimer's disease) at some point prior to study end-point (i.e. on average 4.33 years after entry into the study), giving an average annual conversion rate of $11.4 \%$ (95\% CI 4-23\%). Fifty-nine percent $(26 / 44)$ of participants had not received a clinical

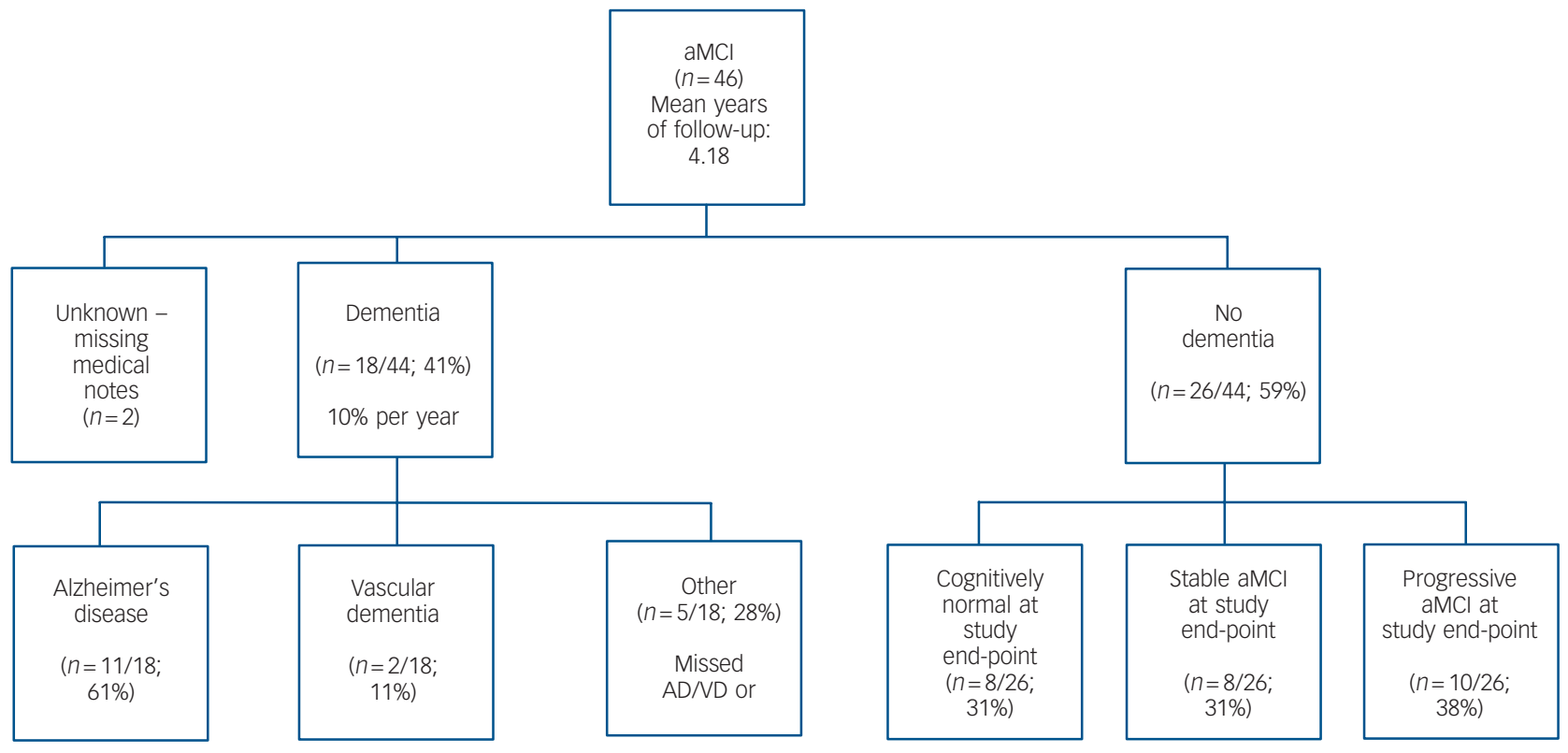

Fig. 1 Flow chart of amnestic mild cognitive impairment (aMCl) end-point classification in accordance with clinical diagnoses in medical notes. 


Table 2 Summary of final regression model ${ }^{\mathrm{a}}$

diagnosis of dementia. Medical notes were missing or not accessible for the remaining 2/44 individuals. For these participants, the most up-to-date information available at the study end-point was that obtained at final study attendance. Of the participants who had not received a clinical diagnosis of dementia, $8 / 26$ (31\%) were stable, $10 / 26(38 \%)$ progressive and $8 / 26(31 \%)$ reverted to normal, according to the criteria defined above (Fig. 1).

Following adjustment of the alpha level, ${ }^{24}$ significant differences in the baseline performances of the aMCI converters group and the aMCI non-converters group were found on the ACE $\left(t_{(42)}=2.98, P<0.01\right)$ and HVLT-R discrimination index $\left(t_{(41)}=2.81, P<0.01\right)$ (Table 1$)$.

Only one participant obtained a GNT score at baseline below the 2 nd centile of our healthy elderly control group. None of the participants with aMCI performed below the 2.5th centile of our control group on the GFT at baseline. Univariate sensitivity, specificity, negative (NPV) and positive (PPV) predictive values for conversion from aMCI to dementia were therefore based on a cut-off performance at the 7 th centile (s.d. $>1.5$ below the mean) of age norms, and can be summarised as follows: GNT sensitivity $38 \%$, specificity 68\%, PPV 43\%, NPV 63\%; GFT $44 \%, 68 \%, 50 \%, 63 \%$. Using a combination cut-off of ACE $<88 / 100$ or PAL $>14$ errors, the overall rate of classification accuracy was $68 \%$, with sensitivity $72 \%$, specificity $65 \%$, PPV $59 \%$ and NPV $77 \%$.

Backward logistic regression with age, NART FSIQ, years of follow-up and the neuropsychological measures for which baseline performance differentiated converters and non-converters (HVLT-R discrimination index and ACE total score) resulted in a final model, completed after five iterations, including the variables ACE total score and HVLT-R discrimination index score only, yielding an overall classification accuracy (aMCI converters group $v$. aMCI non-converters group) of $74 \%$, sensitivity $65 \%$, specificity 80\%, NPV 77\% and PPV 69\% (Table 2).

\section{Discussion}

\section{Main findings}

Forty-one percent of participants who met criteria for aMCI at study entry received a clinical diagnosis of dementia within the following 4 years, giving an annual conversion rate of $10 \%$ which is almost identical to the mean $(9.7 \%)$ annual conversion rate obtained on averaging the findings from existing clinic-based aMCI longitudinal studies of a similar (2.5-3.5 year) length.

Baseline performance on the ACE and HVLT-R discrimination index could discriminate between future aMCI converters and non-converters at a time when general levels of cognitive functioning fell above the higher level screening cut-off for dementia (i.e. $>27 / 30$ on the MMSE and $>88 / 100$ on the $\mathrm{ACE}$ ), and classified these individuals in accordance with their prognostic fate with a moderate degree $(74 \%)$ of overall accuracy. Differences in the baseline performances of the aMCI converters and non-converters groups on these measures could not be explained by differences in age, FSIQ or length of follow-up, as the two groups were roughly similar and effects persisted after controlling for each of these variables.

The average score of the converters group on the HVLT-R discrimination index at baseline was equal to performance at the 4th centile of published age- and education-matched control values, ${ }^{19}$ and the 7 th centile of our own healthy elderly age- and IQ-matched control sample. The corresponding values for the non-converters group were the 28th and the 36th centile, respectively, implying that there is a greater risk of conversion to dementia among a subset of people with aMCI, who are readily identifiable on the basis of published norms.

For the ACE, average scores of the converters group were equal to the 4th, those of non-converters equal to the 31 st centile of published normative values, ${ }^{11}$ and the 1 st and 16th centile of our matched study control data, providing further support for the designation of $88 / 100$ as a higher cut-off point for dementia. We suggest that use of this score is appropriate to screen for aMCI, despite the younger age group of the original ACE normative sample.

\section{Implications}

In clinical practice, the combined performances of people with aMCI on the ACE and HVLT-R discrimination index could be used to inform decisions about the frequency of future contact/ monitoring required, or in combination with additional clinical information (i.e. levels of carer-rated depressive symptoms, ${ }^{28}$ ApoE4 carrier status, ${ }^{15}$ corroborative history, neuroimaging findings, family history and qualitative aspects of clinical presentation) to decide whether or not to consider pharmacological or other interventions. The relatively small proportion of people with aMCI showing resolution of their cognitive symptoms over the time of the study also has implications for the clinical management of such individuals, as a number of empirically validated methods for the cognitive rehabilitation of early-stage Alzheimer's disease have been described ${ }^{29}$ and could theoretically be used to enhance the day-to-day memory functioning of people with aMCI.

Baseline scores of the HVLT-R discrimination index and the ACE were significant independent predictors of conversion to dementia. Closer inspection of the regression analysis reveals that the HVLT-R discrimination index score contributes to the overall classification accuracy of the ACE by increasing negative predictive value. This implies that memory impairment of a consolidation/ storage nature is generally present in individuals where a diagnosis of dementia (Alzheimer's disease, vascular dementia or mixed Alzheimer's disease/vascular dementia) follows within 4 years.

It is possible that cued recall impairment arises closer to the point at which Alzheimer's disease can be diagnosed clinically, often after problems with (the more difficult) free recall become apparent. The possibility that cueing may facilitate episodic recall 
may then disappear with disease progression, giving rise to an encoding/consolidation profile of memory impairment.

This observation has implications for the recently proposed new research criteria for Alzheimer's disease, ${ }^{30}$ in which the requirement for objective evidence of significantly impaired episodic memory has been elaborated. The new criteria emphasise the importance of establishing an encoding and storage deficit on the grounds that reduced benefit from cueing during recall reliably identifies prodromal Alzheimer's disease. Our findings lend support to the specification of episodic memory impairment in this manner. However, the limited range of scores attainable using the HVLT-R discrimination index and the resultant potential for floor effects suggest it may not be well-suited for monitoring significant decline in episodic memory function over time.

The newer version of the ACE- $\mathrm{R}^{31}$ incorporates a delayed cued verbal recognition element. In light of the added predictive value of the HVLT-R discrimination index demonstrated in this study, it would seem prudent to evaluate whether or not this measure retains its prognostic contribution alongside the ACE-R.

The mean total baseline score on the ACE (87/100) for future converters fell just below the higher level cut-off point for dementia. For $26 \%$ of participants, baseline ACE scores fell above the higher cut-off point for dementia (i.e. 88/100), suggesting that where the ACE is used as the sole means of determining the likelihood of developing dementia over the proceeding 4 years, up to a quarter of all individuals with preclinical dementia receive false reassurance of 'normality'. The implications of using the ACE as a sole means to determine the presence or absence of clinically significant levels of cognitive impairment are even greater, as the present findings indicate that $62 \%$ of people who fulfil criteria for aMCI obtain scores of $88 / 100$ or above on the ACE.

We were unable to replicate the high levels of sensitivity, specificity, positive and negative predictive values that have been previously reported in association with combined PAL and ACE scores, the GNT and the GFT. ${ }^{8,10,12}$ Our need to adopt a more conservative 7 th centile (1.5 standard deviation) cut-off for the GFT and GNT naming measures may in part reflect the longer follow-up period in ours as compared with the last study ${ }^{12}$ (13.7 months from baseline until study end-point). Their shorter interval until diagnosis is consistent with a greater magnitude of impairment on naming tasks in their sample. The predictive value of neuropsychological measures is likely to vary as a function of the number of years prior to diagnosis, underscoring the need for careful consideration of both the length of follow-up and the levels of baseline cognitive functioning of aMCI cohorts in different studies.

\section{Limitations}

There are a number of limitations to this study: first, although a mean follow-up period of over 4 years compares well with previous clinic-based studies of longitudinal outcome in people with aMCI, it remains possible that additional participants with aMCI will go on to receive a clinical diagnosis over the longer term. Furthermore, the length of follow-up varied among those with aMCI between 1 and 5 years. Ideally all participants with aMCI would have been followed up for the maximum 5-year interval. Second, the high average premorbid IQ and select nature (i.e. tertiary referral, amnestic single and multidomain and primarily Alzheimer's disease end-point diagnosis) of our aMCI cohort limits generalisation of the study findings beyond groups that are characterised similarly. Third, although the predominant eventual diagnosis of dementia was of Alzheimer $(n=11)$ or mixed $(n=5)$ Alzheimer/vascular type, a small proportion of people with aMCI (i.e. 2) were finally diagnosed with vascular dementia. The resultant inclusion of an end-point clinical diagnosis other than that of pure Alzheimer's disease may have influenced the predictive validity of the neuropsychological measures within our battery. It could be argued, in a more practical sense, that exclusion of people with aMCI on grounds of multiple risk factors or even retrospectively does not reflect clinical reality. There is variability in the point at which clinicians arrive at a diagnosis of dementia and the specific criteria they employ, despite a common bias towards avoiding false-positive diagnoses. It remains possible that for some individuals with an aMCI clinical diagnostic status at the study end-point this was in part reliant on the idiosyncrasies of one or more of the six attending consultants. Finally, the relatively small sample size makes independent replication essential.

Jane A. Lonie, PhD, Mario A. Parra-Rodriguez, MD, Kevin M. Tierney, MSC, Division of Psychiatry, University of Edinburgh; Lucie L. Herrmann, MA, Division of Psychiatry, University of Edinburgh and Department of Psychiatry, University of Oxford; Claire Donaghey, MSc, Division of Psychiatry, University of Edinburgh; Ronan E. O'Carroll, PhD, Department of Psychology, University of Stirling; Klaus P. Ebmeier, MD, Division of Psychiatry, University of Edinburgh and Department of Psychiatry, University of Oxford.

Correspondence: Klaus P. Ebmeier, Department of Psychiatry, University of Oxford, Warneford Hospital, Oxford OX3 7JX, UK. Email: klaus.ebmeier@psych.ox.ac.uk

First received 20 Jan 2010, final revision 18 Mar 2010, accepted 20 Apr 2010

\section{Funding}

Funded by the Gordon Edward Small's Charitable Trust, Edinburgh (Scottish Charity Register: SC008962).

\section{Acknowledgement}

The revised dual task was generously provided by Della Sala and colleagues.

\section{References}

1 Blossom CMS, Matthews FE, McKeith IG, Bond J, Brayne C. Early cognitive change in the general population: how do different definitions work? J Am Geriatr Soc 2007; 55: 1534-40.

2 Chertkow H, Nasreddine Z, Joanette Y, Drolet V, Kirk J, Massoud F, et al. Mild cognitive impairment and cognitive impairment, no dementia: Part A, concept and diagnosis. Alzheimers Dement 2007; 3: 266-82.

3 Visser PJ, Kester A, Jolles J, Verhey F. Ten-year risk of dementia in subjects with mild cognitive impairment. Neurology 2006; 67: 1201-7.

4 Amieva $\mathrm{H}$, Jacqmin-Gadda $\mathrm{H}$, Orgogozo JM, Le Carret $\mathrm{N}$, Helmer $\mathrm{C}$, Letenneur $\mathrm{L}$, et al. The 9 year cognitive decline before dementia of the Alzheimer type: a prospective population-based study. Brain 2005; 128: 1093-101.

5 Fox NC, Warrington EK, Seiffer AL, Agnew SK, Rossor MN. Presymptomatic cognitive deficits in individuals at risk of familial Alzheimer's disease. Brain 1998; 121: 1631-9.

6 Tabert MH, Manly JJ, Liu XH, Pelton GH, Rosenblum S, Jacobs M, et al. Neuropsychological prediction of conversion to Alzheimer disease in patients with mild cognitive impairment. Arch Gen Psychiatry 2006; 63: 916-24.

7 Folstein MF, Folstein SE, McHugh PR. 'Mini-mental state'. A practical method for grading the cognitive state of patients for the clinician. J Psychiatric Res 1975; 12: 189-98

8 Blackwell AD, Sahakian BJ, Vesey R, Semple JM, Robbins TW, Hodges JR. Detecting Dementia: Novel Neuropsychological Markers of Preclinical Alzheimer's Disease. Dement Geriatr Cogn 2004; 17: 42-8.

9 McKenna P, Warrington EK. Graded Naming Test. nferNelson, 1983.

10 Ahmed S, Mitchell J, Arnold R, Nestor PJ, Hodges JR. Predicting rapid clinical progression in amnestic Mild Cognitive Impairment. Dement Geriatr Cogn 2008; 25: 170-7.

11 Mathuranath PS, Nestor PJ, Berrios GE, Rakowicz W, Hodges JR. A brief cognitive test battery to differentiate Alzheimer's disease and frontotemporal dementia. Neurology 2000; 55: 1613-20 
12 Thompson SA, Graham KS, Patterson K, Sahakian BJ, Hodges JR. Is knowledge of famous people disproportionately impaired in patients with early and questionable Alzheimer's disease? Neuropsychology 2002; 16: 344-58

13 Schmand B, Huizenga HM, van Gool WA. Meta-analysis of CSF and MRI biomarkers for detecting preclinical Alzheimer's disease. Psychol Med 2010; 40: $135-45$.

14 Lonie JA, Herrmann LL, Donaghey CL, Ebmeier KP. Clinical referral patterns and cognitive profile in mild cognitive impairment. Br J Psychiatry 2008; 192 59-64.

15 Petersen RC, Thomas RG, Grundman M, Bennett D, Doody R, Ferris S, et al. Vitamin $E$ and donepezil for the treatment of mild cognitive impairment. N Engl J Med 2005; 352: 2379-88

16 Lonie JA, Herrmann LL, Tierney KM, Donaghey C, O'Carroll R, Lee A, et al. Lexical and semantic fluency discrepancy scores in $\mathrm{aMCl}$ and early Alzheimer's disease. J Neuropsychol 2009; 3: 79-92.

17 Lonie JA, Tierney KM, Herrmann LL, Donaghey C, O'Carroll RE, Lee A, et al Dual task performance in early Alzheimer's disease, amnestic mild cognitive impairment and depression. Psychol Med 2009; 39: 23-31.

18 Nelson H, Willison J. National Adult Reading Test (Part II) Test Manual. NFER-Nelson, 1991.

19 Benedict RHB, Schretlen D, Groninger L, Brandt J. Hopkins Verbal Learning Test - Revised: normative data and analysis of inter-form and test-retest reliability. Clin Neuropsychologist 1998; 12: 43-55.

20 Rey A. Psychological examination of a case of post-traumatic encephalopathy. Arch Psychol 1941; 28: 286-340.

21 Reitan RM. Validity of Trail Making Test as an indication of organic brain disease. Percept Mot Skills 1958; 8: 271-6.
22 Della Sala S. Paper and Pencil Dual Task. University of Edinburgh, undated (http://www.psy.ed.ac.uk/people/sdsala/tests/sdsdualtask).

23 Benton, AL, Hamsher K. Multilingual Aphasia Examination. AHA Associates, 1983

24 Holm S. A simple sequential rejective multiple test proceedure. Scand J Stat 1979; 6: 65-70.

25 Mebane-Sims I. Alzheimer's disease facts and figures. Alzheimers Dement 2009: 5: $234-70$

26 Whalley $\mathrm{L}$, Starr JM, Athawes R, Hunter D, Pattie A, Deary IJ. Childhood mental ability and dementia. Neurology 2000; 55: 1455-9.

27 Petersen RC, Smith GE, Waring SC, Ivnik RJ, Kokmen E. Mild Cognitive impairment: clinical characterization and outcome. Arch Neurol 1999; 56: 303-8.

$28 \mathrm{Lu} \mathrm{PH}$, Edland SD, Teng E, Ingus K, Petersen RC, Cummings JL. Donepezil delays progression to $\mathrm{AD}$ in $\mathrm{MCl}$ subjects with depressive symptoms. Neurology 2009: 72: 2115-21.

29 Clare L, Woods RT. Cognitive training and cognitive rehabilitation for people with early-stage AD: a review. Neuropsychol Rehabil 2004; 14: 401.

30 Dubois B, Feldman HH, Jacova C, DeKosky ST, Barberger-Gateau P, Cummings $J$, et al. Reserach criteria for the diagnosis of $A D$ : revising the NINCDS-ADRDA criteria. Lancet Neurol 2007; 6: 734-46.

31 Mioshi E, Dawson K, Mitchell J, Arnold R, Hodges JR. The Addenbrooke's Cognitive Examination Revised (ACE-R): a brief cognitive test battery for dementia screening. Int J Geriatr Psychiatry 2006; 21: 1078-85.

\section{psychiatry}

\section{Of course it is (the delusion that's really true)}

\section{Peter Byrne}

William Burroughs described the paranoid man as one 'who knows a little of what's going on'. In that rare beast, a mainstream Hollywood film that portrays schizophrenia with humanity and without a murder, A Beautiful Mind (2001), John Nash (Russell Crowe) irritates his wife when he says he heard the garbage truck outside at night. He has been hospitalised with psychosis and in that movie convention much imitated in life, anything he says must be taken as fantasy, unless proven otherwise. But the garbage guys are outside and thus begins a process where she (and the audience) begin to trust and identify with Nash again. This is the exception that proves the rule. When a filmic character with mental illness reports the 'unfortunate event' on which the film turns, nobody believes him/her: The Couch Trip (1988), Twelve Monkeys (1995), Independence Day (1996), Conspiracy Theory (1997) and K-Pax (2001) all milk this conceit for its full comic potential. Director Alan J. Pakula's paranoid trilogy Klute (1971), The Parallax View (1974) and All the President's Men (1975) project the angst of the unbelieved onto a battered American audience, reeling from Vietnam and Nixon. A flavour of paranoia excites modern science fiction (Total Recall, 1990 and the Matrix trilogy, 1999-2003), and infuses the contemporary celebrity film, The Truman Show (1998).

Melodramas take the 'unbelievable mental patient' a stage further. Gaslight was a popular drama (filmed twice: 1940, 1944), where a scheming husband tries to convince his dutiful wife that she is losing her mind by making things go bump in the night. The hero-journalist of Shock Corridor (1963) feigns psychosis to gain admission to the asylum. He identifies the murderer but no one believes him; his continued incarceration drives him insane. The parents of Claudia Draper in Nuts (1987) conspire to undermine her testimony against a murder charge. Despite her histrionics, Claudia (Barbara Streisand) wins out. Her battle with a bullying psychiatrist is mirrored in The Changeling (2008). A woman's child goes missing but the Los Angeles police return the wrong boy. To avoid further embarrassment to the police, an unscrupulous psychiatrist declares her insane.

Where A Beautiful Mind succeeds is in drawing in the audience into seeing and believing the content of Nash's delusions. By the time the film demarcates reality from illness, we have been seduced by his experiences, and we feel his confusion and loss. True empathy. 